\title{
Quantification of the Hysteresis of Macroscopic Fundamental Diagrams and Its Relationship with the Congestion Heterogeneity and Performance of a Multimodal Network
}

\author{
Seham Hemdan' ${ }^{1}$ Amr M. Wahaballa ${ }^{2,3}$, Fumitaka Kurauchi ${ }^{3 *}$ \\ ${ }^{1}$ Graduate School of Engineering, Gifu University, Gifu, Japan \\ ${ }^{2}$ Department of Civil Engineering, Aswan University, Aswan, Egypt \\ ${ }^{3}$ Department of Civil Engineering, Gifu University, Gifu, Japan \\ Email: u3812014@edu.gifu-u.ac.jp,amr.whbala@aswu.edu.eg, „kurauchi@gifu-u.ac.jp
}

How to cite this paper: Hemdan, S., Wahaballa, A.M. and Kurauchi, F. (2018) Quantification of the Hysteresis of Macroscopic Fundamental Diagrams and Its Relationship with the Congestion Heterogeneity and Performance of a Multimodal Network. Journal of Transportation Technologies, 8, 44-64.

https://doi.org/10.4236/jtts.2018.81003

Received: November 28, 2017

Accepted: December 25, 2017

Published: December 28, 2017

Copyright $\odot 2018$ by authors and Scientific Research Publishing Inc. This work is licensed under the Creative Commons Attribution International License (CC BY 4.0).

http://creativecommons.org/licenses/by/4.0/

\begin{abstract}
Recent studies have observed hysteresis loops in the macroscopic fundamental diagram (MFD). In particular, for the same network density, higher network flows occur during congestion onset than during congestion offset. To evaluate management strategies using the MFD, investigating the relationship between the size of these loops and network performance is needed. The existing literature has mainly discussed correlating loop width (difference in density) and height (capacity drop) with congestion heterogeneity, but has failed to prove a relationship between the capacity drop and traffic conditions. Moreover, quantification of the MFD loop in complex multimodal networks has not been investigated. The objective of this paper covers these aspects. We simulated the Sioux Falls network with different mode-share ratios (car and bus users) based on a multi-agent simulation, MATSim. We investigated the relationships between MFD loop size and congestion heterogeneity (standard deviation of density) and network performance (average passenger travel time), and found that both were directly correlated with loop width, while weakly correlated with loop height. Moreover, we divided the MFD loop into two parts according to congestion onset and offset periods and found that the heights of the two parts had opposite effects. Accordingly, we show why the relationship between capacity drop and congestion heterogeneity is not found in the literature. We also found that network performance inversely affected the height of part of the loop while the height of its other part increased with an increase in congestion heterogeneity. These results help to evaluate network performance in the presence of MFD hysteresis, leading to elaborated management decisions.
\end{abstract}




\section{Keywords}

Macroscopic Fundamental Diagram, Hysteresis Loop, Congestion

Heterogeneity, Multimodal, Network Performance

\section{Introduction}

Traffic congestion is a severe matter worldwide as it causes delays and air pollution. Thus, several traffic-management strategies have been developed to control traffic congestion. However, evaluating the efficiency of any traffic management strategy prior to its implementation is a challenge due to the wide variety of control variables that make the joint optimization of all traffic-flow measures difficult. This is, for instance, due to the large data sets that need to be processed and the large solution space needed. Alternatively, the macroscopic fundamental diagram (MFD), proposed by [1] and [2], may have the potential to provide a simpler way to assess traffic control impacts on transportation networks. MFD represents a network's traffic operations by a scatter plot relating the average network density and flow over a certain period. Instead of a detailed description of the traffic state, one variable, the number of vehicles in an area, may help to describe the traffic state. Geroliminis and Daganzo [2] showed that the concept worked in practice for road traffic in the center of a major city. It was claimed, though, that the concept of the MFD worked only for homogeneous traffic conditions.

Buisson and Ladier [3] used empirical observations of different freeways and showed that the MFD may have a significant amount of scatter; that is, multiple flows may be observed for a given value of density, and the flows during the onset of congestion differ significantly from those during the dissipation of congestion (later referred to as hysteresis). Mazloumian et al. [4] found similar MFD hysteresis loops in a simulation of an urban grid network. They found, for different estimations of spatial distribution of congestion (represented by the standard deviation of the densities), that the MFD had different shapes. Subsequently, many researchers (e.g., [5] [6] [7] [8]) have confirmed the presence of MFD hysteresis loops using empirical data and simulation results. Gayah and Daganzo [8] tested the hysteresis on MFD in a simplified two-bin network and concluded that the existence of a MFD hysteresis loop can be expected even on a symmetric network with uniform demand. Thus, in real-world conditions of city-scale networks, a hysteretic type of MFD may exist. Qian [9] simulated installing ramp meters to restrict vehicle entry to a highway when its flow reached a certain threshold. Qian concluded that hysteresis did not affect recognition of the patterns of a MFD and that the critical density and the maximum flow could be recognized readily. Nevertheless, few research efforts have been made concerning the relationship between MFD hysteresis loops and traffic conditions, as detailed in Section 2.3. Hemdan et al. [10] used MFD to qualify the effect of route, 
mode, and departure-time choices on multimodal network performance based on simulation results. However, a quantitative evaluation of MFD loops was not provided. Quantifying MFD hysteresis loops and correlating them to different traffic characteristics may help in comparing the effects of different management strategies on network performance, leading to more appropriate management decisions.

Recently, Saberi and Mahmassani [11], using loop detector data on freeways, found that the heterogeneous spatial distribution of network occupancy was independent of both the width of a hysteresis loop (difference in density) and its height (capacity drop); however, it was correlated with the area of the hysteresis loop. Shi and Lin [12] used loop detector data from urban expressways and found that the mean speed correlated with the width of the hysteresis loop. However, consistent with the results of [11], it was not correlated with capacity drop. Orfanou et al. [13] correlated capacity drop with hysteresis loop shape based on vehicle trajectory data. They also found that the capacity drop could not fully describe the form or duration of the hysteresis loop. These studies and others tried to illustrate the main characteristics of MFD hysteresis loops; however, they failed in interpreting the loose relationship between capacity drops and the spatial heterogeneity of density. In this study, we continue the previous work on quantifying and characterizing MFD hysteresis loops. We attempted to find a relationship between loop height/width and the heterogeneity of congestion over the network. Additionally, the hysteresis loop was correlated with a network performance indicator, represented by the average passenger travel time consumed. We illustrate the reasons why capacity drop appearing in the MFD loop and spatial heterogeneity of density are insignificantly correlated in the literature and how they can be correlated.

The next section reviews the relevant literature with an overview of using MFDs as an indicator of network performance under different traffic control strategies. Section 3 illustrates the simulation tool used in this paper and the development of MFDs for different network characteristics and travel choices. Section 4 presents the results of the proposed quantification of the MFD hysteresis loop and its relationships. Section 5, finally, presents a summary and conclusions.

\section{Literature Review}

The MFD, developed earlier by [14] and reintroduced by [1] [2] [15] [16] is a representation of a network's traffic operations by a scatter plot for the average flow on a network and its average density. Because our focus is on comparing network performance under different control strategies in the presence of a MFD hysteresis loop, the next subsections summarize the relevant research.

\subsection{Control Strategy Evaluation Based on MFDs}

MFDs have been used to represent traffic evolution and to evaluate network 
performance. Geroliminis and Daganzo [2], using data collected from loop detectors and GPS-equipped taxis in Yokohama, proved the existence of a MFD for a city network. Later, many researchers investigated MFDs for urban networks and freeways, based on simulations (e.g., [17] [18] [19]), and empirical data (e.g., [2] [3] [6] [20]).

MFDs have been used to evaluate the performance of transport networks subject to a zone-based routing system and cordon pricing (e.g., [21] and [22]). Zheng et al. [23] studied a time-dependent area-based pricing and have updated pricing value based on the critical density appear in MFD. Their focus was considering users heterogeneity and the traveler's behavioral adaptation to the pricing values. Zhang et al. [24] studied the impacts of various adaptive traffic signaling methods on signalized intersections with two-way arterial streets, while [19] performed a simulation on an idealized grid network. They found that adaptive traffic signals showed consistent MFDs when the network was moderately congested. Ji et al. [17] evaluated the effect of providing four ramp meters in one direction on the "A10 West" highway, Amsterdam. They found that ramp metering had a direct effect on MFD shape. The reduced inflow resulted in higher flows and lower accumulation with ramp metering, whereas without ramp metering, accumulation increased significantly while flow decreased (resulting in heavy congestion). Qian [9] simulated installing ramp meters on urban roads feeding the A10 highway to restrict vehicle feeding when the flow on the A10 reached a certain threshold. It was found that, for the A10 highway, the maximum density during the congestion period decreased, while the maximum flow value remained the same. For urban roads, congestion branches did not appear on the MFD before the implementation of ramp metering. However, the curve with ramp metering showed flow reduction at high densities, and the maximum density increased. Qian concluded that hysteresis did not restrict recognizing MFD patterns and that important values, such as the critical density and the maximum flow, could be recognized readily from MFDs. He suggested that the size of the drop in MFD loops when congestion resolved (the difference between the values of flow accompanying a certain value of density) could indicate the severity of congestion.

Other researchers (e.g., [25] [26] [27]) have used MFDs to analyze multimodal network performance. Zheng and Geroliminis [25] used MFDs to evaluate the effects of road space distribution on the performance of a congested multimodal network by optimization and simulation. The objective of their optimization was to minimize the total passenger hours traveled (PHT) by reallocating road space among modes. A simulation was performed on a bi-modal city case study to investigate the performance under a dynamic space allocation strategy. They concluded that PHT could be minimized by a dynamic space allocation strategy that used the bus lane space better during the off-peak period and served more passengers during the peak period. Geroliminis et al. [27] developed a three-dimensional MFD (3D-MFD) for a bi-modal network based on simulation 
data. They related the number of cars and buses to the total flow in the network and found that the network's vehicle flow decreased monotonically with the number of buses serving in the network, whereas passenger throughput was maximized at a non-zero accumulation of buses.

In summary, analyses using MFDs for evaluating the effects of different traffic management strategies suggest that networks can be evaluated based on this approach. According to changes in critical density and maximum flow values, the performance of networks can be qualified under different traffic management strategies. Additionally, the size of the drop in MFD hysteresis loops when congestion resolves may reflect congestion severity.

\subsection{Hysteresis Phenomena}

Early investigation of hysteresis phenomena belongs to [28] and [29]. They illustrated the hysteresis of the speed-density relationship according to acceleration and deceleration. Detailed illustration of traffic hysteresis characteristics can be found in [30]. Recently, Ji et al. [17] performed a simulation of a combination of an urban arterial and an urban freeway and found that during congestion onset and offset, the MFD was scattered. Buisson and Ladier [3] used empirical data on Toulouse and showed that the MFD might have a hysteresis. They summarized the following possible reasons for such hysteresis:

- Differences between highways, surface streets, city center, and suburban roads.

- When demand is not distributed uniformly, congestion may occur in some parts of the network.

- The appearance and disappearance of congestion can also be a reason for successive values of mean flow.

- Differences in data-measurement locations: some loops are located near traffic signals, and some are much farther away.

For urban networks, Mazloumian et al. [4] showed by simulation that the variance in density over different locations was an important factor when determining total network performance. They concluded that, "An inhomogeneity in the spatial distribution of car density increases the probability of spillover, which substantially decreases the network flow." Later, Geroliminis and Sun [6] confirmed the findings of [4] using empirical data from the Yokohama metropolitan area. Daganzo et al. [7] showed that turning at intersections was among the key reasons for the drop in the performance with unevenly spread congestion. Gayah and Daganzo [8] tested the effects of driver adaptivity on MFD hysteresis for a homogeneous square-grid network simplified as a two-bin network. They concluded that, when congestion resolved, it did not do so instantaneously at all locations; however, it did resolve completely from one side of the queue, causing clockwise hysteresis loops in the MFD. They also concluded that these clockwise hysteresis loops should be expected, even under conditions of perfect network symmetry with uniform demand, and that they will diminish when drivers avoid 
congestion by an adaptive re-routing process. They suggested that the presence of highly adaptive drivers might explain why no hysteresis loop existed in Yokohama, and they expected the Yokohama network to exhibit a clockwise hysteresis loop if it was subjected to disturbances. Knoop et al. [21] concluded that congestion resolution would increase the spatial variance of the accumulation and thus (relatively) decrease the performance. To consider the spatial inhomogeneity, [31] proposed a generalized MFD (GMFD) that related the average flow to both the average density and the inhomogeneity of density (the standard deviation of the densities in different links). They showed that weighted flow was a continuous function of the weighted density and the inhomogeneity of density and they described hysteresis patterns using this function. Their results were consistent with many other studies (e.g., [4]) and MFDs may have a scatter in which multiple flows may be observed for a given value of density.

In response to the appearance of MFD hysteresis loops, some researchers had developed clustering algorithms to divide the network into homogeneous sub-networks to get low scattered MFD (e.g., [32] [33]). Overview of relevant research can be found in [34]. Qian [9] suggested that the size of the drop in MFD loops when congestion resolves (the difference between the values of flow accompanying a certain value of density) could reflect congestion severity. Additionally, [21] concluded that the hysteresis loops in a MFD were an effect by themselves. Nevertheless, there has been limited research concerning the relationship between MFD hysteresis loops and traffic conditions, as detailed below.

\subsection{Characterization of MFD Hysteresis Loops}

Saberi and Mahmassani [20] investigated the effect of the spatial and temporal distribution of congestion on the form and characteristics of MFD hysteresis loops based on loop detector data for Portland and found a scattered pattern in the MFD when the spatial distribution of link densities was inhomogeneous. Also, [8] characterized hysteresis loops by their direction of formation: "clockwise", "counter-clockwise”, and "figure-eight". Later, [11] investigated hysteresis and capacity drop phenomena in three different freeway networks in the US using loop detector data. They identified two types of capacity drop: capacity drop while the network was loading, due to the network's inability to sustain its throughput at its peak value for a relatively long time; and capacity drop associated with network instability during a reloading process such as the afternoon loading period after an incomplete recovery from the morning peak period. They observed two loop shapes on both freeways. A loop was associated with unstable recovery of a network in which the average network flow constantly decreased when the average network occupancy decreased. Another loop shape was observed when the network was recovering, and the average network flow remained roughly unchanged when the average network occupancy decreased. They tried to correlate the size of the hysteresis loop and the standard deviation of network occupancy during the recovery phase. They found a very weak cor- 
relation between the standard deviation of network occupancy and both the width and height of the hysteresis loop. However, they found that the hysteresis loop area correlated with a congestion homogeneity index (the difference between the number of congested and uncongested links divided by the total number of links in the network) with a correlation coefficient of 0.77 . Shi and Lin [12] used loop detector data to investigate the characteristics of MFDs for an urban expressway network in Shanghai, China. They explored factors that affected the characteristics of hysteresis loops. Using two days' data, resulting in four different hysteresis loops, they found a direct correlation between the width of the hysteresis loop and the mean speed, meaning that when congestion of the network was more severe, wider hysteresis loops formed. However, they also found no correlation between the capacity drop of a hysteresis loop and the mean speed.

Although these studies ([11] and [12]) used different variables (standard deviation of network occupancy and mean speed, respectively), there was a direct inverse relationship between these variables when looking at a specific regime (loading or recovery period). In the loading process, the flow and density increased rapidly, the standard deviation of density was relatively low, and the average speed was relatively high (congestion had not happened). When the congestion dissipated, the densities of many links decreased. However, some high-density links, still in congestion, led to higher standard deviation of density among links. Moreover, flows on high-density links were low with congestion and the flows of low-density links were definitely low. Meanwhile, the average network speed in the recovery period was lower because of congestion, which also led to lower network flow. This means that both studies reflect the same result, that capacity drop was independent of the traffic situation.

Orfanou et al. [13] correlated capacity drop, acceleration, and spacing to hysteresis shape based on vehicle trajectory data observed by video cameras on the I-80 freeway in San Francisco. They identified determinants that affected the shape of hysteresis and related them to two driver-behavior classes: aggressive and timid. They classified the loops into two categories and concluded that aggressive behavior by the following vehicle resulted in a counter-clockwise hysteresis loop, whereas timid behavior resulted in a clockwise hysteresis loop. The spacing and acceleration of the following vehicle at the end of the hysteresis were found to be dominant, while the capacity drop between the onset and offset of hysteresis was the least influential factor and could not fully describe the hysteresis loop in terms of its form or duration. Recently, [35] used MFDs to compare traffic performance on various idealized hierarchical urban networks consisting of local and arterial streets using microsimulation. They differentiated arterials from local streets by additional travel lanes and additional green time at intersections. Using the size and shape of hysteresis loops, they found that the presence of arterials had a significant impact on the spatial distribution of congestion in the network. They subjectively compared networks based on the width and 
height of hysteresis loops (a superior network has a smaller width and height). However, they did not investigate the quantitative relationship between the size of hysteresis loops and congestion inhomogeneity.

Like MFD hysteresis loop, [36] proved the existence of a hysteresis loop in the relationship between average travel time and its variance (for journeys with the same average travel time, the variance for departures as congestion dissipates are higher than the variance for departures during the onset of congestion). Few research efforts studied the relationship between MFD loops and travel time variability loops. Gayah et al. [37] and Yildirimoglu et al. [38] studied the average travel time and its day-to-day variability relationship and analyzed the observed hysteresis loops. However, they did not investigate the relationship between the sizes of the two loops. Some promising correlations have found by [39]. They found strong correlations between loops sizes and their components, specifically the differences in flow, density, and the average and standard deviation of the travel time.

In summary, this review shows that characterizing MFD hysteresis loops and their relationships to traffic characteristics is still an ongoing research topic. Few methodologies have been employed and different assumptions have been made regarding the formation of hysteresis loops and the factors that influence them. Little attention has been paid, however, to quantifying MFD hysteresis loops and their relation with congestion inhomogeneity, network performance, and other traffic characteristics. Those who have attempted to do so have used limited sample sizes and studied specific loop shapes and have found, in the main, relationships between loop size and heterogeneity, not network performance. Additionally, the literature has provided no relation between the capacity drop on hysteresis loops and traffic conditions. Moreover, quantifying hysteresis loops in complex multimodal networks has not been investigated. Closing these gaps in the literature is the objective of this paper.

\section{Developing MFDs for Different Multimodal Network Characteristics and Mode Choices}

\subsection{Simulation of a Multimodal Network}

Vehicle and passenger travel behavior can be estimated based on the MATSim framework, which uses an iterative approach for agent-based dynamic traffic assignment. Integrated simulation of private and public transport based on a queuing model allows time-dependent calculation of travel times, accounting for spillover effects and the direct interaction of private and public transport. Agents alter their behavior from iteration to iteration, based on a co-evolutionary algorithm to try to find optimal routes, modes, and departure times and, thus, maximize the total utility of their daily activity schedule. Following each iteration of the queue-based network assignment, the choices of each agent are evaluated and scored, allowing agents to select more successful options for execution of their schedule in the next iteration. The selection of travel alternatives 
from the choice set of each agent is performed based on a random utility model, which, after a number of iterations, leads to the convergence of individual and total utilities and, thus, to an agent-based SUE [40]. The extended Sioux Falls network proposed by [41] was used. The MATSim simulation was performed using the integrated public transport simulation, in which buses share road space with cars and are affected by the congestion. Individual mode choices for agents can be explored and used for MFD drawing.

The extended Sioux Falls network [41] consists of two road types; urban and highway roads, with a total of 334 links and 282 nodes. The bus network consists of five bus lines with a 5-min headway operation. In total, 56,904 workers commute, and 27,206 persons perform secondary activities, with a total of 168,220 daily trips. Every agent performs only one round trip per day (home - work home or home - secondary - home). The time window for an activity and its typical duration affect how peaked or spread out the demand is in the peak-hours, and this will reflect the degree of congestion in the network. The work activity opens at 8 a.m. and closes at 6 p.m., with a typical duration of $9 \mathrm{~h}$ (latest work start time is 9 a.m.), while secondary trips can be taken between 8 a.m. and 8 p.m. and completed within $1 \mathrm{~h}$ during this period. MATSim allows different travel choices for individuals. The simulation presented here enables mode choices for some individuals according to the different traffic management strategies described in the next subsection.

\subsection{Characteristics of Multimodal Network and Mode-Choice Scenarios}

We tested different supply characteristics and different mode-choice combinations to consider a variety of MFD loops. The supply characteristics of scenarios 1, 2, and 3, shown in Table 1, represent homogeneous networks where all links have the same number of lanes, with three different capacity levels. Different mode-choice combinations are developed in Scenarios 4, 5, and 6. The simulation framework presented here enables mode choices for some individuals: some agents can change between using a car, bus, or walking. In this work, we considered only two modes: cars and buses. We simulated a whole day period from

Table 1. Scenarios of different network characteristics and different mode-choice combinations (Sioux Falls network).

\begin{tabular}{|c|c|c|c|c|c|c|c|c|c|c|}
\hline \multirow{3}{*}{ Scenario } & \multirow{3}{*}{ Link type } & \multicolumn{6}{|c|}{ Network characteristics } & \multicolumn{3}{|c|}{ Mode-share ratio } \\
\hline & & \multicolumn{2}{|c|}{ No. of lanes } & \multicolumn{2}{|c|}{ Free flow speed $\mathrm{km} / \mathrm{h}$} & \multicolumn{2}{|c|}{ Flow capacity veh/h } & \multirow{2}{*}{ Car \% } & \multirow{2}{*}{ Bus \% } & \multirow{2}{*}{ walk\% } \\
\hline & & Urban & Highway & Urban & Highway & Urban & Highway & & & \\
\hline Scenario 1 & Urban & 2 & ---- & 50 & ---- & 2000 & ---- & $78 \%$ & $19 \%$ & $3 \%$ \\
\hline Scenario 2 & Urban & 2 & ----- & 50 & ----- & 1500 & ----- & $78 \%$ & $19 \%$ & $3 \%$ \\
\hline Scenario 4 & Urban + Highway & 2 & 3 & 50 & 90 & $800-1000$ & $1700-1900$ & $58 \%$ & $34 \%$ & $8 \%$ \\
\hline Scenario 5 & Urban + Highway & 2 & 3 & 50 & 90 & $800-1000$ & $1700-1900$ & $55 \%$ & $36 \%$ & $8 \%$ \\
\hline Scenario 6 & Urban + Highway & 2 & 3 & 50 & 90 & $800-1000$ & $1700-1900$ & $52 \%$ & $39 \%$ & $9 \%$ \\
\hline
\end{tabular}


12:00 a.m. to 11:59 p.m. Mode-choice rate was controlled by changing the disutility for traveling by car. The marginal disutility for the bus mode was set by referring to [41] and the marginal disutility for the car was varied between 0 and -1 . The different mode-share ratios are shown in scenarios 4, 5, and 6 (Table 1 ). Rieser et al. [42] concluded that less flow fluctuation and a more stable and faster convergence process could be achieved by allowing only some of the population to generate new plans. He stated that a typical set-up in MATSim is to allow re-planning for $10 \%$ of the agents. Mode choice re-planning probabilities were thus permitted for $10 \%$ of agents in this paper, and agent selections were based on agent-based SUE, as illustrated in Section 3.1. Bus fleet size and headway remained constant for all cases. The MFD represents the relationship between the average network flow and the average network density. The MFDs for different scenarios are shown in Figure 1. We used the MATSim outputs to calculate the values of the average network flow, the average network density, the standard deviation of density among links and the average passenger travel time using the following equations.

$$
\begin{aligned}
& Q_{t}=\frac{\sum_{i \in z} q_{i, t} l_{i}}{\sum_{i \in z} l_{i}} \\
& K_{t}=\frac{\sum_{i \in z} k_{i, t} l_{i}}{\sum_{i \in z} l_{i}} \\
& \sigma_{t}=\sqrt{\frac{\sum_{i \in z}\left(l_{i}\left(k_{i, t}-\bar{K}_{t}\right)^{2}\right)}{\sum_{i \in z} l_{i}}} \\
& \bar{\sigma}_{\text {hys }}=\sqrt{\frac{\sum_{t \in h y s} \sum_{i \in z}\left(l_{i}\left(k_{i, t}-\bar{K}_{t}\right)^{2}\right)}{\sum_{i \in z} l_{i}}} \\
& T T_{t}=\sum_{j \in D T_{t}} T_{j} \\
& \overline{T T}_{h y s}=\frac{\sum_{t \in h y s} \sum_{j \in D T_{t}} T_{j}}{\sum_{j \in D T_{t}} D T_{t}}
\end{aligned}
$$

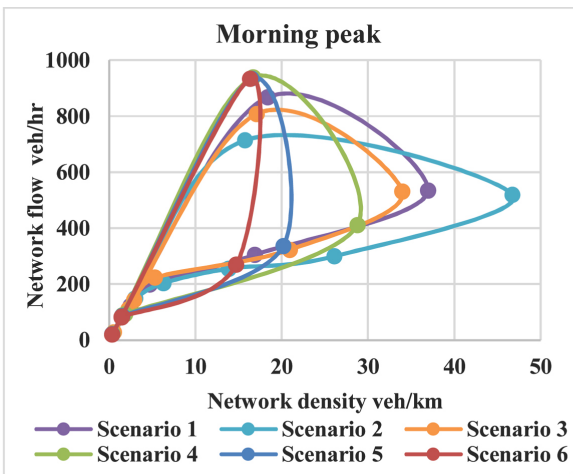

(a)

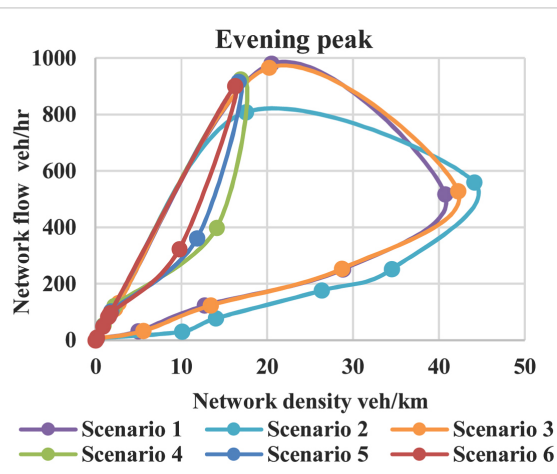

(b)

Figure 1. MFDs for different scenarios (a) morning peak, (b) evening peak. 
where,

$Q_{t}$ : Weighted average of network flow at time $t(\mathrm{veh} / \mathrm{h})$.

$K_{t}$ : Weighted average of network density at time $t(\mathrm{veh} / \mathrm{km})$.

$q_{i, t}:$ Flow of link $i$ at time $t$.

$k_{i, t}$ : Density of link $i$ at time $t$.

$l_{i}$ : Length of link $i$.

$z$ : Set of links in the network.

$\bar{k}_{t}$ : Average of link densities at time $t$.

$\sigma_{t}:$ Standard deviation of density at time $t$.

$\bar{\sigma}_{\text {hys }}$ : Average of standard deviation of density over a hysteresis loop.

$T_{j}$ : Trip travel time of traveler $j(\mathrm{~min})$.

$D T_{t}$ : Set of traveler whose departure time is $t$.

$\overline{T T}_{h y s}$ : Average passenger travel time over a hysteresis loop.

\section{Quantification of a MFD Loop and Its Relationship with Density Spatial Heterogeneity}

\subsection{Quantification of a MFD Loop}

Based on [11] and [12], we represented hysteresis loops by width (difference in density), height (capacity drop), and area. Moreover, we decomposed the loops into congestion onset and offset times (associated with the maximum flow and loop start points). The reason for doing so was derived from the important results found by [43]. They developed an automatic calculation method to extract hysteresis loops from network flow-density data by looking at loop transitions, which are constituted by the maximum average network flow, start and end points of the hysteresis loop, and its direction. Their results showed higher spatial spreads of density during the starting point of the hysteresis loop. Thus, we suggest here that looking at these transition points may help in answering the question of why the effect of capacity drop has not been found in the literature; it may have existed at some interval but not have been detected when looking at overall loop intervals. Thus, in the next subsections, we will analyze different transition points and suggest dividing loop area into two parts (Figure 2). We will also test the relationship considering both loading and recovery periods instead of looking at recovery period alone, as [11] did.

\subsection{Relationship between Loop Metrics and Standard Deviation of Density}

Previous research approximated the size of a hysteresis loop as the product of its width and height. To ensure the accuracy of this approximation, we calculated the actual size of the loop for a random two-thirds sample of MFDs shown in Figure 1 (four morning and four evening loops) and compared the results with the approximation. As shown in Figure 3, a reasonable coefficient of determination $\left(R^{2}\right)$ value found between the actual area and its approximation, when correlated with the standard deviation of density, the approximated area size 


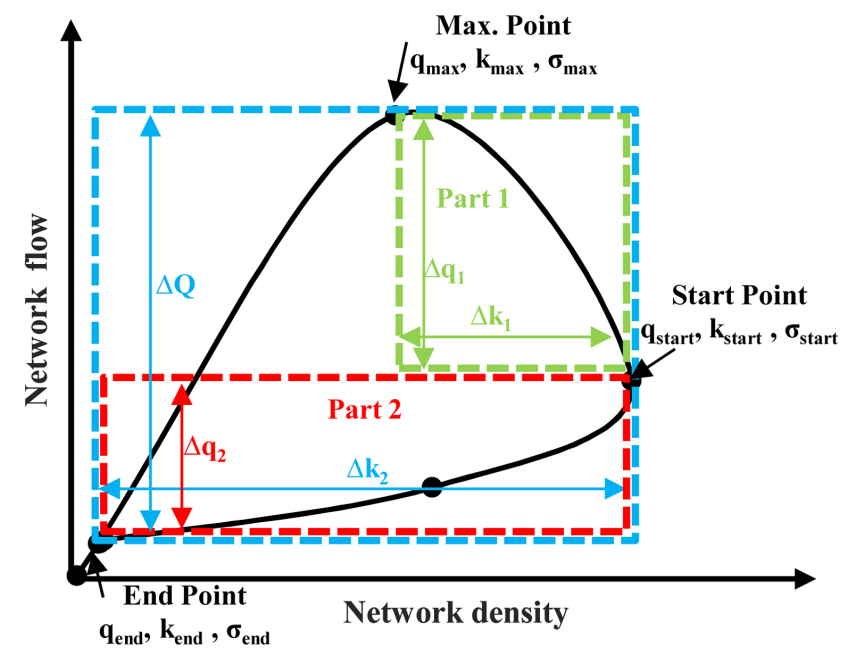

Figure 2. Transition points on MFD loops.

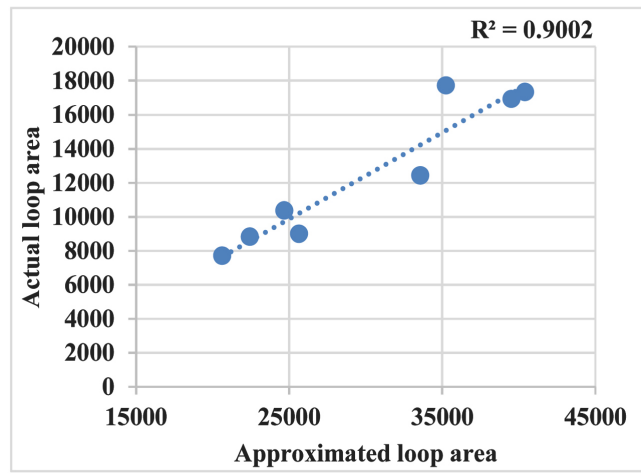

(a)

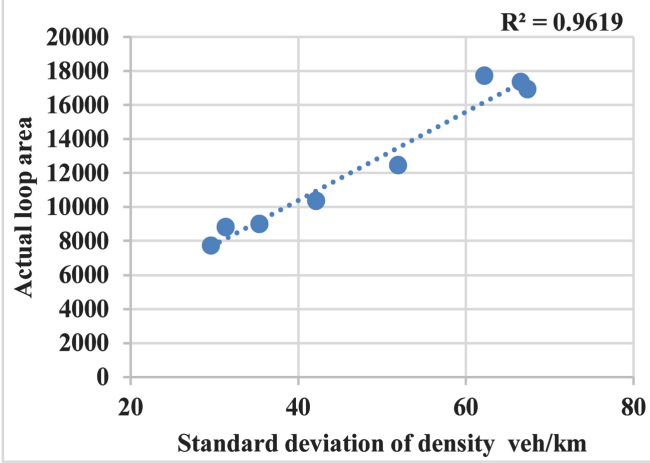

(b)

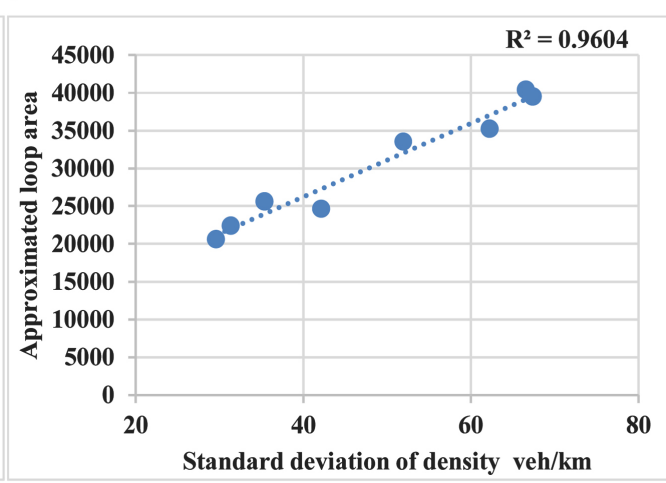

(c)

Figure 3. Correlation between (a) actual versus approximated loop area, (b) the standard deviation of density versus actual loop area, and (c) the standard deviation of density versus approximated loop area.

showed almost the same correlation trend and accuracy as with the actual one. Thus, for simplicity, we determined the next set of relationships using the approximated area size (by multiplying the width and height of the loop). Different relationships were found between the average standard deviation of density (for the recovery phase only and considering both the loading and recovery phases) and loop metrics. Table 2 shows the $R^{2}$ values for those relationships. 
Table 2. $R^{2}$ values for relations between standard deviation of density and loop metrics.

\begin{tabular}{ccccccccccc}
\hline & \multicolumn{3}{c}{ Whole loop } & \multicolumn{3}{c}{ Part 1 loop } & \multicolumn{3}{c}{ Part 2 loop } \\
\cline { 2 - 9 } & $\left(\Delta q^{\star} \Delta k\right)$ & $\Delta k$ & $\Delta q$ & $\left(\Delta q_{1}^{*} \Delta k_{1}\right)$ & $\Delta k_{1}$ & $\Delta q_{1}$ & $\left(\Delta q_{2}^{*} \Delta k\right)$ & $\Delta k$ & $\Delta q_{2}$ \\
\hline $\begin{array}{c}\text { Average standard } \\
\text { deviation of density } \\
\text { (calculated over } \\
\text { recovery phase only) }\end{array}$ & 0.762 & 0.634 & 0.046 & 0.554 & 0.570 & -0.320 & 0.862 & 0.634 & 0.841 \\
$\begin{array}{c}\text { Average standard } \\
\text { deviation of density } \\
\text { (calculated over } \\
\text { loading and } \\
\text { recovery phases) }\end{array}$ & 0.784 & 0.721 & 0.021 & 0.578 & 0.646 & -0.390 & 0.902 & 0.721 & 0.857 \\
\hline
\end{tabular}

where

Max point: point at maximum flow.

Start point: point at loop start.

End point: point at loop ends.

Whole loop: loop with height $\Delta q$ and width $\Delta k$.

Part 1 loop: congested part with height $\Delta q_{1}$ and width $\Delta k_{1}$.

Part 2 loop: recovery part with height $\Delta q_{2}$ and width $\Delta k$.

Judging from Table 2, the standard deviation of density is directly correlated with the whole loop area and its width. Considering loading and recovery periods improved the correlation between the standard deviation of density and loop area and its width. For the whole loop area, the $R^{2}$ value when the average standard deviation of density was calculated over the recovery period only, was 0.762 . However, when the average standard deviation of density was calculated over loading and recovery periods, the $R^{2}$ value was 0.784 . Additionally, a weak correlation was found between loop height and standard deviation of density (as in the results of [11] [12] [13]). Although the overall loop area correlation to the standard deviation of density may be sufficient, dividing the loop into two parts provides additional insights:

- The correlation between the standard deviation of density and the metrics of the Part 2 loop are stronger than for the overall loop. That is, the Part 2 loop alone can represent the heterogeneity effect.

- The correlation between the standard deviation of density and the heights of two loop parts $\left(R^{2}=0.390\right.$ and 0.857 for $\Delta q_{1}$ and $\Delta q_{2}$, respectively) are stronger than its correlation with overall loop height $\left(R^{2}=0.021\right)$. Additionally, the two heights are oppositely correlated to the standard deviation of density (Figure 4). This illustrates why the relationship between capacity drop and congestion inhomogenity was not found in previous research ([11] [12] [13]). There may be two reasons:

1) $\Delta q_{1}$ is the capacity drop that occurred while demand was still high and the network was loading, and is associated with the inability of the network to sustain its throughput at its maximum value for a long time [11]. During a congestion regime, more loading leads to less heterogeneity because all links are congested; 


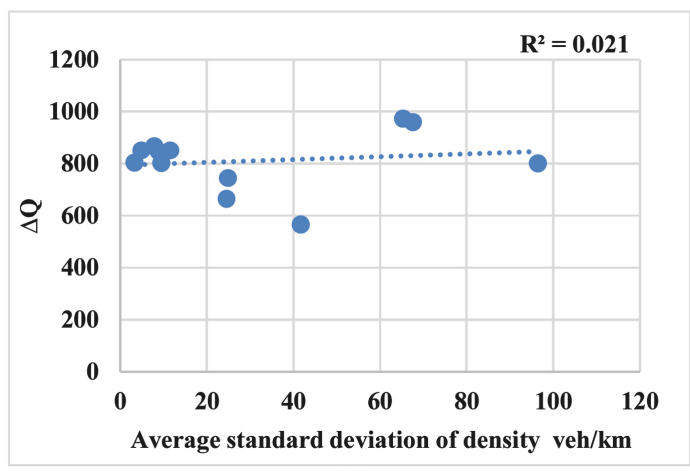

(a)

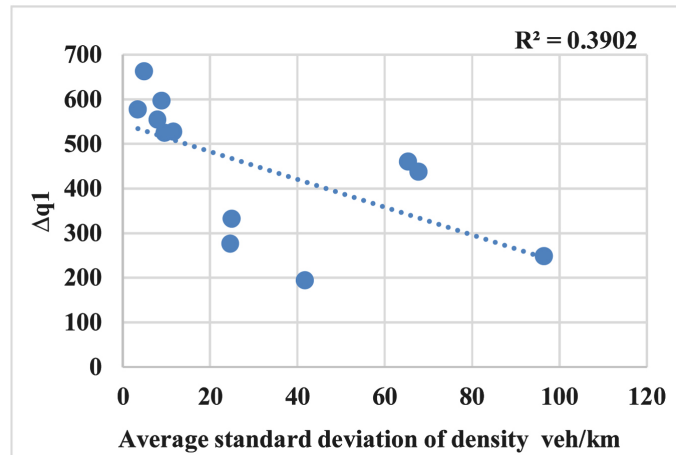

(b)

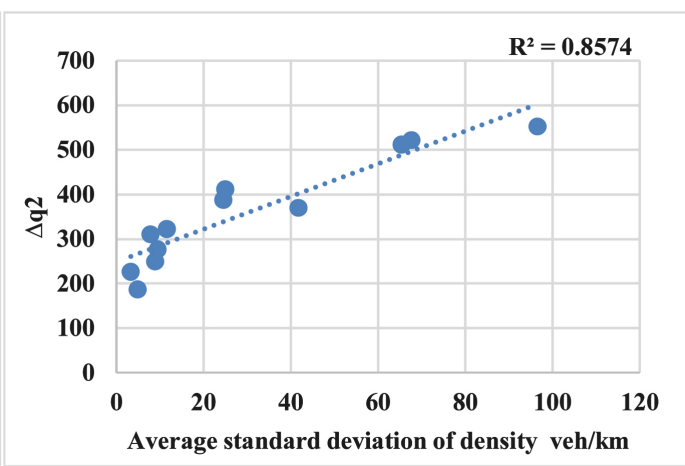

(c)

Figure 4. Relationship between average standard deviation of density with (a) height of overall loop (b) height of part 1 loop and (c) height of part 2 loop.

this increased congestion causes a decrease in network flow and, consequently, an increase in $\Delta q_{1}$. Thus, $\Delta q_{1}$ increases with the decrease in standard deviation of density.

2) $\Delta q_{2}$ occurred during the recovery process. During congestion dissipation, the densities of many links will decrease; however, some links are still in congestion, leading to a higher standard deviation of density among links. The flow on most links will decrease (due to congestion or due to low link occupancy) and, consequently, $\Delta q_{2}$ will increase. Thus, the larger is $\Delta q_{2}$, the more heterogeneous is the congestion distribution during the recovery phase.

\subsection{Relationship between Loop Metrics and Network Performance}

To compare a wide range of mode-share ratios and, consequently, a variety of network performance conditions, the scenarios generated by [10], as shown in Table 3, were used here. The scenarios were developed by varying the marginal disutility for the car between 1.0 and -1.0 and leaving everything else constant, allowing mode choice re-planning for $10 \%$ of the agents, as illustrated in Section 3.2. Note that bus fleet size and headway were constant in all cases. Figure 5 represents the relationship between the average network flow and the average network density for different mode choice combinations during the morning and evening peaks. The relationship between the size of a MFD loop and network 
Table 3. Mode-choice ratio for different scenarios according to different car disutility $(\beta c)[10]$.

\begin{tabular}{ccccccc}
\hline & \multicolumn{5}{c}{ Car disutility $(\beta c)$} \\
\cline { 2 - 7 } & Base case & 1 & 0.5 & 0 & -0.5 & -1 \\
\hline Bus \% & $19 \%$ & $29 \%$ & $30 \%$ & $34 \%$ & $36 \%$ & $39 \%$ \\
Car \% & $78 \%$ & $65 \%$ & $62 \%$ & $58 \%$ & $55 \%$ & $52 \%$ \\
Walking \% & $3 \%$ & $6 \%$ & $7 \%$ & $8 \%$ & $8 \%$ & $9 \%$ \\
\hline
\end{tabular}

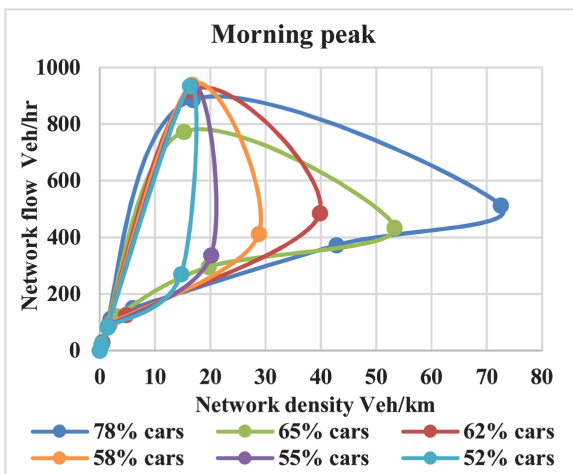

(a)

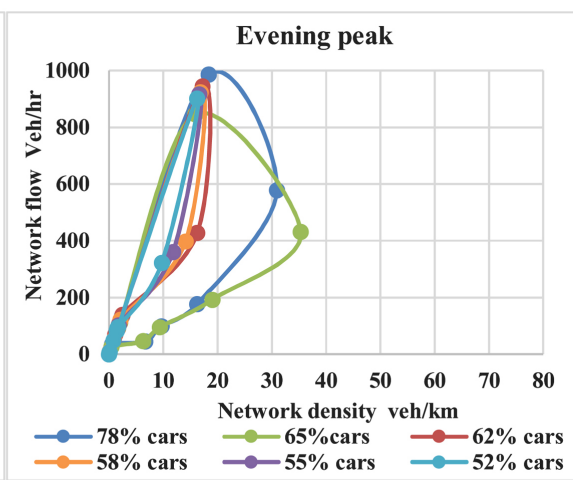

(b)

Figure 5. MFDs for different mode-share ratios (a) morning peak (b) evening peak [10].

performance (measured by the average passenger travel time) was tested.

Table 4 shows $R^{2}$ values for the relationships between the average passenger travel time and loop metrics. A direct correlation was found between the average passenger travel time and loop size and width. Also, a weak relationship was found between the average passenger travel time and overall loop height, which is the same result as the relationship between the standard deviation of density and whole loop height (Table 2). However, by dividing the loop into two parts, a clear inverse relationship was found between the average passenger travel time and the height of Part 1 of the loop $\left(R^{2}=0.701\right)$. However, a weak correlation was found between the average passenger travel time and the height of Part 2 of the loop $\left(R^{2}=0.510\right)$ (Figure 6). Comparing Figure 4 and Figure 6 shows that the correlation between the standard deviation of density and the height of the Part 2 loop is stronger than its correlation with the height of the Part 1 loop, whereas the correlation between the average passenger travel time and the height of the Part 1 loop is stronger than its correlation with the height of the Part 2 loop. This means that network performance inversely affects the height of the Part 1 loop whereas the height of the Part 2 loop increases with the increase of the spatial spread of density. Network performance inversely affects the capacity drop while the network is loading, while the inhomogeneous spatial distribution of congestion increases the flow reduction during network unloading with congestion dissipation. As discussed in detail in Section 4.2, the capacity drop in the Part 1 loop occurs due to network loading, although it is unable to sustain its flow at its maximum value for long. More loading leads to a decrease in network 
Table 4. Summary of $R^{2}$ values for relationships between network performance and loop metrics.

\begin{tabular}{ccccccccccc}
\hline & \multicolumn{3}{c}{ Whole loop } & \multicolumn{3}{c}{ Part 1 loop } & \multicolumn{4}{c}{ Part 2 loop } \\
\cline { 2 - 9 } & $\left(\Delta q^{\star} \Delta k\right)$ & $\Delta k$ & $\Delta q$ & $\left(\Delta q_{1}^{*} \Delta k_{1}\right)$ & $\Delta k_{1}$ & $\Delta q_{1}$ & $\left(\Delta q_{2}^{*} \Delta k\right)$ & $\Delta k$ & $\Delta q_{2}$ \\
\hline $\begin{array}{c}\text { Average } \\
\text { passenger } \\
\text { travel time }\end{array}$ & 0.860 & 0.883 & 0.336 & 0.767 & 0.806 & -0.701 & 0.888 & 0.883 & 0.510 \\
\hline
\end{tabular}

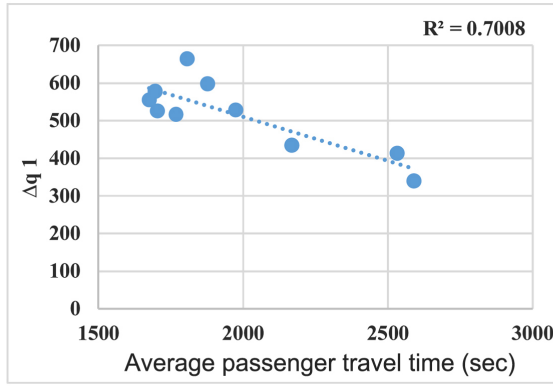

(a)

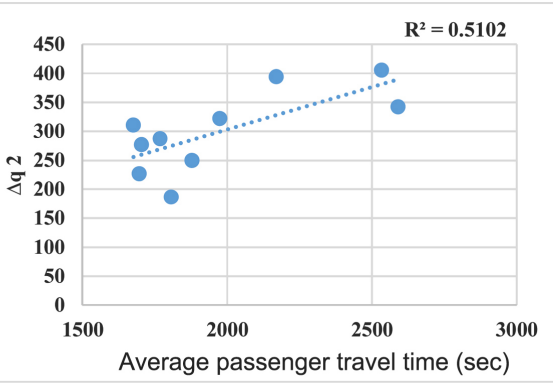

(b)

Figure 6. Relationship between average passenger travel time and (a) height of part 1 loop and (b) height of part 2 loop.

flow correlated with an increase in $\Delta q_{1}$. While $\Delta q_{2}$ occurs during congestion dissipation, as some link densities decrease and some links are still in congestion, this leads to a higher standard deviation of density among links that is correlated with the $\Delta q_{2}$ increase.

From Figure 5, increasing the number of car users increases the density and severity of congestion, as indicated by the increase in the width of the MFD loop. The scenarios of "78\% Cars" and "65\% Cars" (where 78\% and 65\% of travelers use cars, respectively) lead to more severe congestion and larger $\Delta k_{1}$. Consequently, a higher average passenger travel time occurs. However, $\Delta q_{1}$ decreases according to its inverse relationship to average passenger travel time; this is accompanied by a decrease in traffic flow and a large increase in density (large $\Delta k_{1}$ ). This increase in density can be eliminated by reducing the number of car users. The scenario of " $58 \%$ Cars" results in light congestion, indicated by a decrease in traffic flow and a small increase in density, accompanied by an increase in $\Delta q_{1}$ and, consequently, a lower average passenger travel time. An additional decrease in the percentage of car users, while keeping the bus fleet the same, would allow the network to operate with nearly free flow: see the results for the “ $52 \%$ Cars" and " $55 \%$ Cars" modes.

\section{Summary and Conclusions}

MFDs that relate the average network density and flow have been used in the literature for network performance evaluation. However, it is difficult to attain low scattered MFDs in real-world city-scale networks: instead, recent studies have suggested more complex MFDs that exhibit a hysteresis loop. In particular, for the same network density, a higher network flow occurs during congestion 
onset than during congestion offset. Investigating the relationship between these loop sizes and network performance is of great importance in comparing the effect of different management strategies. This study investigated quantifying MFD hysteresis loops and the relationship between the size of this loop and both congestion heterogeneity and network performance in multimodal networks.

We estimated MFDs for traffic conditions associated with different mode-share ratios (car and bus users) on the Sioux Falls network, based on a multi-agent transport simulation. Mode choice was based on a random utility model with an agent-based stochastic user equilibrium. The goal was to compare traffic conditions associated with different mode-share ratios, based on MFDs, and, consequently, evaluate the effect of different management strategies on network performance in the presence of a hysteresis loop. First, we evaluated a quantification method for MFD loops. Previous research [11] and [12] has approximated hysteresis loop area as the product of its width and height. To ensure that this approximation does not affect result accuracy, we calculated the actual loops' areas for a sample of the developed MFDs and compared the results with the approximation. Second, we correlated loop dimensions with the standard deviation of density (representing congestion heterogeneity) and the average passenger travel time (as an indicator of multimodal network performance). Moreover, we decomposed the problem into its components by dividing loop area into two parts, according to congestion onset and offset times (associated with the maximum flow point and loop start points during network recovery). We tested the effect of partitioning loop area, based on the important results found by [43], who concluded that a higher spatial spread of density occurred during the starting point of the hysteresis loop.

By correlating with the standard deviation of density, the approximated area showed almost the same correlation trend and accuracy as did the actual area. We conclude that a hysteresis loop's width (difference in density), its height (capacity drop), and its area (by multiplying the loop's width and height) are representative metrics for MFD loops. Different relationships were found between the average standard deviation of density and loop metrics. It is concluded that the average standard deviation of density should be calculated over both loading and recovery periods, and not for the recovery period alone, especially for loops covering a long period. Dividing loop area into two parts according to congestion onset and offset times (upper and lower parts, respectively) provides some important insights. We consider, to some extent, that the partitioning criteria for a loop's area suggested in this paper are helpful for understanding a number of relationships that have not been found in the literature. It was found that the correlation between the standard deviation of density and the loop's lower part is stronger than its correlation with the overall loop. That is, the lower part of the loop alone can represent the heterogeneity effect. We also found that the correlation between the standard deviation of density and the heights of both loops' parts was stronger than the correlation with overall loop height. This is because the two heights have opposite effects on the standard deviation of den- 
sity, which affects the correlation with the overall loop height. This illustrates why no relationship between capacity drop and congestion inhomogeneity has been found in previous research ([11] [12] [13]), as interpreted in detail in Section 4.2 .

For network performance, a clear inverse relationship was found between the average passenger travel time and the height of the upper part of the loop $\left(R^{2}=\right.$ $0.70)$, while it was weakly correlated with the height of the lower part of the loop $\left(R^{2}=0.51\right)$. Additionally, useful conclusions were reached when comparing the correlation between the metrics of the MFD loop suggested in this paper and both the standard deviation of density and the average passenger travel time. It was found that the standard deviation of density was strongly and directly correlated with the height of the lower part of the loop while the average passenger travel time had a strong inverse correlation with the height of the upper part of the loop. We conclude that the height of the upper part of the loop is sensitive to the average passenger travel time whereas the height of the lower part of the loop is more sensitive to the standard deviation of density. That is, network performance inversely affects the capacity drop while the network is loading, whereas the inhomogeneous spatial distribution of congestion increases the flow reduction during network unloading with congestion dissipation. These findings may help in using the hysteric MFDs that are expected for city-scale networks. This may provide a simple tool for comparing the effects of management strategies on network performance, and contribute to more elaborate decisions. Further statistical analysis would benefit from a larger sample of hysteric MFDs to test properly for the representational power and generalization ability of the developed relationships.

\section{References}

[1] Daganzo, C.F. (2007) Urban Gridlock: Macroscopic Modeling and Mitigation Approaches. Transportation Research Part B: Methodological, 41, 49-62. https://doi.org/10.1016/j.trb.2006.03.001

[2] Geroliminis, N. and Daganzo, C.F. (2008) Existence of Urban-Scale Macroscopic Fundamental Diagrams: Some Experimental Findings. Transportation Research Part B: Methodological, 42, 759-770. https://doi.org/10.1016/j.trb.2008.02.002

[3] Buisson, C. and Ladier, C. (2009) Exploring the Impact of Homogeneity of Traffic Measurements on the Existence of Macroscopic Fundamental Diagrams. Transportation Research Record: Journal of the Transportation Research Board, 2124, 127-136. https://doi.org/10.3141/2124-12

[4] Mazloumian, A., Geroliminis, N. and Helbing, D. (2010) The Spatial Variability of Vehicle Densities as Determinant of Urban Network Capacity. Philosophical Transactions of the Royal Society A: Mathematical, Physical and Engineering Sciences, 368, 4627-4647. https://doi.org/10.1098/rsta.2010.0099

[5] Geroliminis, N. and Sun, J. (2011) Hysteresis Phenomena of a Macroscopic Fundamental Diagram in Freeway Networks. Transportation Research Part A: Policy and Practice, 45, 966-979. https://doi.org/10.1016/j.tra.2011.04.004

[6] Geroliminis, N. and Sun, J. (2011) Properties of a Well-Defined Macroscopic Fun- 
damental Diagram for Urban Traffic. Transportation Research Part B: Methodological, 45, 605-617. https://doi.org/10.1016/j.trb.2010.11.004

[7] Daganzo, C.F., Gayah, V.V. and Gonzales, E.J. (2011) Macroscopic Relations of Urban Traffic Variables: Bifurcations, Multivaluedness and Instability. Transportation Research Part B: Methodological, 45, 278-288.

https://doi.org/10.1016/j.trb.2010.06.006

[8] Gayah, V.V. and Daganzo, C.F. (2011) Clockwise Hysteresis Loops in the Macroscopic Fundamental Diagram: An Effect of Network Instability. Transportation Research Part B: Methodological, 45, 643-655.

https://doi.org/10.1016/j.trb.2010.11.006

[9] Qian, X. (2009) Application of Macroscopic Fundamental Diagrams to Dynamic Traffic Management. M.Sc. Thesis, Delft University of Technology.

[10] Hemdan, S., Wahaballa, A.M. and Kurauchi, F. (2017) Evaluating Travel Choices Effect on Multimodal Network Performance using Vehicle and Passenger Macroscopic Fundamental Diagrams. Journal of the Eastern Asia Society for Transportation Studies.

[11] Saberi, M. and Mahmassani, H.S. (2013) Empirical Characterization and Interpretation of Hysteresis and Capacity Drop Phenomena in Freeway Networks. Transportation Research Record: Journal of the Transportation Research Board, 2391, 44-55. https://doi.org/10.3141/2391-05

[12] Shi, X. and Lin, H. (2014) Exploring the Characteristics of Hysteresis Phenomena of Macroscopic Fundamental Diagram for Urban Expressway Network: The Case of Shanghai. Transportation Research Board 93rd Annual Meeting, Washington DC, No. 14-2060.

[13] Orfanou, F., Vlahogianni, E. and Karlaftis, M. (2012) Identifying Features of Traffic Hysteresis on Freeways. Transportation Research Board 91 st Annual Meeting, Washington DC, No. 12-1587.

[14] Godfrey, J.W. (1969) The Mechanism of a Road Network. Traffic Engineering and Control, 11, 323-327.

[15] Geroliminis, N. and Daganzo, C.F. (2007) Macroscopic Modeling of Traffic in Cities. Transportation Research Board 86th Annual Meeting, Washington DC, No. 7-413.

[16] Daganzo, C.F. and Geroliminis, N. (2008) An Analytical Approximation for the Macroscopic Fundamental Diagram of Urban Traffic. Transportation Research Part B: Methodological, 42, 771-781. https://doi.org/10.1016/j.trb.2008.06.008

[17] Ji, Y., Daamen, W., Hoogendoorn, S., Hoogendoorn-Lanser, S. and Qian, X. (2010) Investigating the Shape of the Macroscopic Fundamental Diagram using Simulation Data. Transportation Research Record: Journal of the Transportation Research Board, 2161, 40-48. https://doi.org/10.3141/2161-05

[18] Gonzales, E., Chavis, C., Li, Y. and Daganzo, C. (2011) Multimodal Transport in Nairobi, Kenya: Insights and Recommendations with a Macroscopic Evidence-Based Model. Transportation Research Board 90 th Annual Meeting, Washington DC, No. 11-3045.

[19] Gayah, V.V., Gao, X. and Nagle, A.S. (2014) On the Impacts of Locally Adaptive Signal Control on Urban Network Stability and the Macroscopic Fundamental Diagram. Transportation Research Part B: Methodological, 70, 255-268. https://doi.org/10.1016/j.trb.2014.09.010

[20] Saberi, M. and Mahmassani, H.S. (2012) Exploring Properties of Networkwide Flow-Density Relations in a Freeway Network. Transportation Research Record: 
Journal of Transportation Research Board, 2315, 153-163. https://doi.org/10.3141/2315-16

[21] Knoop, V., Hoogendoorn, S. and Van Lint, J. (2012) Routing Strategies Based on Macroscopic Fundamental Diagram. Transportation Research Record: Journal of the Transportation Research Board, 2315, 1-10. https://doi.org/10.3141/2315-01

[22] Zheng, N., Waraich, R.A., Axhausen, K.W. and Geroliminis, N. (2012) A Dynamic Cordon Pricing Scheme Combining the Macroscopic Fundamental Diagram and an Agent-Based Traffic Model. Transportation Research Part A: Policy and Practice, 46, 1291-1303. https://doi.org/10.1016/j.tra.2012.05.006

[23] Zheng, N., Rérat, G. and Geroliminis, N. (2016) Time-Dependent Area-Based Pricing for Multimodal Systems with Heterogeneous Users in an Agent-Based Environment. Transportation Research Part C: Emerging Technologies, 62, 133-148. https://doi.org/10.1016/j.trc.2015.10.015

[24] Zhang, L., Garoni, T.M. and de Gier, J. (2013) A Comparative Study of Macroscopic Fundamental Diagrams of Arterial Road Networks Governed by Adaptive Traffic Signal Systems. Transportation Research Part B: Methodological, 49, 1-23. https://doi.org/10.1016/j.trb.2012.12.002

[25] Zheng, N. and Geroliminis, N. (2013) On the Distribution of Urban Road Space for Multimodal Congested Networks. Transportation Research Part B: Methodological, 57, 326-341. https://doi.org/10.1016/j.trb.2013.06.003

[26] Xie, X., Chiabaut, N. and Leclercq, L. (2013) Macroscopic Fundamental Diagram for Urban Streets and Mixed Traffic. Transportation Research Record: Journal of the Transportation Research Board, 2390, 1-10. https://doi.org/10.3141/2390-01

[27] Geroliminis, N., Zheng, N. and Ampountolas, K. (2014) A Three-Dimensional Macroscopic Fundamental Diagram for Mixed Bi-Modal Urban Networks. Transportation Research Part C: Emerging Technologies, 42, 168-181. https://doi.org/10.1016/j.trc.2014.03.004

[28] Edie, L. (1963) Discussion of Traffic Stream Measurements and Definitions. 2nd International Symposium on the Theory of Traffic Flow, London, 25-27 June 1963, 139-154.

[29] Treiterer, J. and Myers, J. (1974) The Hysteresis Phenomenon in Traffic Flow. Transportation and Traffic Theory, 6, 13-38.

[30] Zhang, H.M. (1999) A Mathematical Theory of Traffic Hysteresis. Transportation Research Part B: Methodological, 33, 1-23. https://doi.org/10.1016/S0191-2615(98)00022-8

[31] Knoop, V., Hoogendoorn, S. and Van Lint, J. (2013) The Impact of Traffic Dynamics on the Macroscopic Fundamental Diagram. Transportation Research Board 92nd Annual Meeting, Washington DC, No. 13-0595.

[32] Ji, Y. and Geroliminis, N. (2011) Spatial and Temporal Analysis of Congestion in Urban Transportation Networks. Transportation Research Board 90 th Annual Meeting, Washington DC, No. 11-1808.

[33] Ji, Y. and Geroliminis, N. (2012) On the Spatial Partitioning of Urban Transportation Networks. Transportation Research Part B: Methodological, 46, 1639-1656. https://doi.org/10.1016/j.trb.2012.08.005

[34] Jain, A.K. (2010) Data Clustering: 50 Years beyond K-Means. Pattern Recognition Letters, 31, 651-666. https://doi.org/10.1016/j.patrec.2009.09.011

[35] Muhlich, N., Gayah, V.V. and Menendez, M. (2015) An Examination of MFD Hysteresis Patterns for Hierarchical Urban Street Networks using Micro-Simulation. 
Transportation Research Record: Journal of the Transportation Research Board, 2491, 117-126. https://doi.org/10.3141/2491-13

[36] Fosgerau, M. (2010) On the Relation between the Mean and Variance of Delay in Dynamic Queues with Random Capacity and Demand. Journal of Economic Dynamics and Control, 34, 598-603. https://doi.org/10.1016/j.jedc.2009.12.002

[37] Gayah, V.V., Dixit, V.V. and Guler, S.I. (2014) Relationship between Mean and Day-to-Day Variation in Travel Time in Urban Networks. EURO Journal on Transportation and Logistics, 3, 227-243. https://doi.org/10.1007/s13676-013-0032-2

[38] Yildirimoglu, M., Limniati, Y. and Geroliminis, N. (2015) Investigating Empirical Implications of Hysteresis in Day-to-Day Travel Time Variability. Transportation Research Part C: Emerging Technologies, 55, 340-350.

https://doi.org/10.1016/j.trc.2015.03.012

[39] Wahaballa, A.M. and Kurauchi, F. (2018) Correlation between the Hysteresis of Travel Time Variability and Macroscopic Fundamental Diagrams on a Bimodal Network. 7 th International Symposium on Transport Network Reliability, INSTR Proceedings.

[40] Nagel, K. and Flötteröd, G. (2009) Agent-Based Traffic Assignment: Going from Trips to Behavioral Travelers. Proceedings International Conference on Travel Behavior Research, Jaipur, 1-26.

[41] Chakirov, A. and Fourie, P.J. (2014) Enriched Sioux Falls Scenario with Dynamic and Disaggregate Demand. Technical Report, Future Cities Laboratory, Singapore ETH Centre (SEC).

[42] Rieser, M., Grether, D. and Nagel, K. (2009) Adding Mode Choice to Multiagent Transport Simulation. Transportation Research Record: Journal of the Transportation Research Board, 2132, 50-58. https://doi.org/10.3141/2132-06

[43] Xu, Z., Jin, P.J., Zhang, J. and Ran, B. (2014) An Automatic Calculation Method of Identifying the Hysteresis Loop Characteristics in Macroscopic Fundamental Diagram. Transportation Research Board 93rd Annual Meeting, Washington DC, No. 14-2305. 